\title{
Sens public
}

\section{Facebook. L’école des Fans}

\section{Gérard Wormser}

Facebook. L’école des fans

URI : https://id.erudit.org/iderudit/1059059ar

DOI : https://doi.org/10.7202/1059059ar

Aller au sommaire du numéro

Éditeur(s)

Département des littératures de langue française

ISSN

2104-3272 (numérique)

Découvrir la revue

Citer ce document

Wormser, G. (2018). Facebook. L'école des Fans. Sens public. https://doi.org/10.7202/1059059ar

\section{Résumé de l'article}

Dans L’Ecole des fans, Gérard Wormser questionne les dimensions communicationnelles des disruptions contemporaines. Nos immenses possibilités d'accès aux échanges et aux savoirs contrastent avec les frustrations qui se développent au cœur des collectivités humaines - plus techniques et plus inégales que jamais. La connectivité universelle s'accompagne de ruptures brutales des liens d'intégration traditionnels. Elle abolit les médiations publiques au profit d'un mixte combinant des possibilités neuves à un isolement croissant. Soumis aux pressions qui nous emportent, comme l'indiquent leurs retombées aux USA ou au Brésil, les réseaux alimentent des régressions fantasmatiques et la peur de l'Autre. Au terme d'un parcours décrivant les manières dont nos réseaux associent la toute-puissance de l'argent à des chocs anthropologiques, Gérard Wormser rejoint les intuitions de Simmel qui avait perçu cette question voici plus d'un siècle.
Creative Commons Attribution-ShareAlike 4.0 International (CC BY-SA 4.0) Sens Public, 2018
Ce document est protégé par la loi sur le droit d'auteur. L'utilisation des services d'Érudit (y compris la reproduction) est assujettie à sa politique d'utilisation que vous pouvez consulter en ligne.

https://apropos.erudit.org/fr/usagers/politique-dutilisation/ 


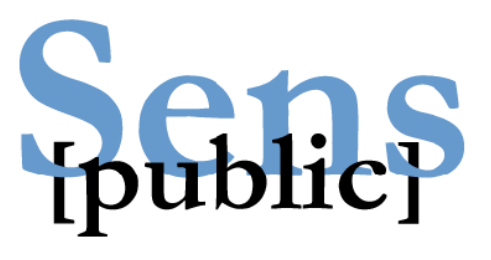

\title{
Facebook. L'école des Fans
}

\author{
Gérard Wormser
}

Publié le 19-10-2018

http://sens-public.org/article1357.html

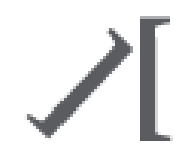

Creative Commons Attribution-ShareAlike 4.0 International (CC BY-SA 4.0) 


\title{
Résumé
}

Dans L'Ecole des fans, Gérard Wormser questionne les dimensions communicationnelles des disruptions contemporaines. Nos immenses possibilités d'accès aux échanges et aux savoirs contrastent avec les frustrations qui se développent au cœur des collectivités humaines plus techniques et plus inégales que jamais. La connectivité universelle s'accompagne de ruptures brutales des liens d'intégration traditionnels. Elle abolit les médiations publiques au profit d'un mixte combinant des possibilités neuves à un isolement croissant. Soumis aux pressions qui nous emportent, comme l'indiquent leurs retombées aux USA ou au Brésil, les réseaux alimentent des régressions fantasmatiques et la peur de l'Autre. Au terme d'un parcours décrivant les manières dont nos réseaux associent la toute-puissance de l'argent à des chocs anthropologiques, Gérard Wormser rejoint les intuitions de Simmel qui avait perçu cette question voici plus d'un siècle.

\begin{abstract}
In L'Ecole des Fans, Gérard Wormser questions the communicative dimensions of contemporary disruptions. The immense opportunities of trade and knowledge access contrast with the frustrations that are growing in human communities - which are more technical and unequal than ever before. Universal connectivity comes with abrupt breaks in traditional integration links. It abolishes public mediation in favour of a mixture of new possibilities and increasing isolation. Subjected to the pressures that prevail, as their fallout in the US or Brazil shows, the networks fuel fantasy regressions and fear of the Other. At the end of a journey describing the ways in which our networks associate the omnipotence of money with anthropological shocks, Gérard Wormser joins Simmel's intuitions, who had perceived this question more than a century ago.
\end{abstract}

Mots-clés : Facebook, Zuckerberg, Sartre, liberté, éthique de l'information

Keywords: Facebook, Zuckerberg, Sartre, freedom, ethics of information 


\section{Table des matières}

$\begin{array}{ll}\text { Introduction } & 4\end{array}$

Sommaire du dossier $\quad 5$

Cynisme et succès . . . . . . . . . . . . . . . . . . 5

Engagement etnconsentement . . . . . . . . . . . 5 


\title{
Facebook. L'école des Fans
}

\author{
Gérard Wormser
}

\section{Introduction}

L'Ecole des fans aborde les dimensions communicationnelles des disruptions contemporaines. Nos immenses possibilités d'accès aux échanges et aux savoirs contrastent avec les frustrations qui se développent au cœur des collectivités humaines - plus techniques et plus inégales que jamais. La connectivité universelle s'accompagne de ruptures brutales des liens d'intégration traditionnels. Elle abolit les médiations publiques au profit d'un mixte combinant des possibilités neuves à un isolement croissant.

Soumis aux pressions qui nous emportent, comme l'indiquent leurs retombées aux USA ou au Brésil, les réseaux alimentent des régressions fantasmatiques et la peur de l'Autre. Au terme d'un parcours décrivant les manières dont nos réseaux associent la toute-puissance de l'argent à des chocs anthropologiques, Gérard Wormser rejoint les intuitions de Simmel qui avait perçu cette question voici plus d'un siècle.

L'auteur suit au plus près la manière dont Mark Zuckerberg et ses proches surfent sur la vague qu'ils ont créée, car la croissance non maittrisée de Facebook est un événement mondial aux effets managériaux et éditoriaux majeurs. Ses aspects touchent aussi bien notre vie quotidienne que la politique internationale, et les penser exige de passer par une enquête philosophique. 


\section{Sommaire du dossier}

Note sur la présente édition : En 2010, la publication du texte "Banco!... L'espace public à l'heure du numérique" dans la revue Sens public, marquait le début de la réflexion de Gérard Wormser sur les enjeux politiques et philosophiques de la culture numérique. Près de dix ans plus tard, la revue Sens public rassemble dans un dossier spécial, préfacé par Pierre Lévy, les principales contributions de l'auteur sur ces questions, parues depuis une dizaine d'année dans différentes revues, auxquelles s'ajoutent des textes inédits.

Ces textes font l'objet d'une publication monographique, disponible en impression à la demande, aux Ateliers de [sens public].

- Pierre Lévy, Préface

\section{Cynisme et succès}

— Gérard Wormser, Banco !... L'espace public à l'heure du numérique

- Comprendre les ressorts de l'évolution des sociétés de masse : À propos de Faire l'histoire de Christophe Bouton et de Who Owns the Future? de Jaron Lanier (texte publié en 2014 dans la Revue française de science politique, vol. 64 / 3, avec le titre « Comprendre et agir sur les ressorts de l'évolution des sociétés de masse », p. 507-512)

- Mark Zuckerberg et la tentation hégémonique a été publié sur Sens public en 2017 avec le titre « Building Global Community : La tentation hégémonique de Mark Zuckerberg et de Facebook ».

- Facebook et la crise des élites a été publié sur Sens public en 2017 avec le même titre.

\section{Engagement etnconsentement}

- Les accrocs d'une stratégie mondiale

- Les Conquérants : Comment les plateformes colonisent l'espace attentionnel

- La raison pratique de la société des réseaux

- Postace : De l'authenticité au pratico-inerte 